\title{
Multiple ionization and fragmentation dynamics of molecular iodine studied in IR-XUV pump-probe experiments
}

\author{
K. Schnorr, ${ }^{\text {a }}$ A. Senftleben, ${ }^{\text {ab }}$ G. Schmid, ${ }^{\text {a }}$ A. Rudenko, ${ }^{c}$ M. Kurka, ${ }^{\text {}}$ \\ K. Meyer, ${ }^{a}$ L. Foucar, ${ }^{d}$ M. Kübel, ${ }^{\text {ef }}$ M. F. Kling, ${ }^{\text {ef }}$ Y. H. Jiang, ${ }^{\mathrm{g}}$ S. Düsterer, ${ }^{\text {h }}$ \\ R. Treusch, ${ }^{h}$ C. D. Schröter, ${ }^{a}$ J. Ullrich, ${ }^{\text {ai }}$ T. Pfeifer ${ }^{a}$ and R. Moshammer ${ }^{a}$
}

Received 4th March 2014, Accepted 14th April 2014

DOI: $10.1039 / c 4 f d 00031 e$

The ionization and fragmentation dynamics of iodine molecules $\left(\mathrm{I}_{2}\right)$ are traced using very intense ( $10^{14} \mathrm{~W} \mathrm{~cm}^{-2}$ ) ultra-short ( $60 \mathrm{fs}$ ) light pulses with $87 \mathrm{eV}$ photons of the Freeelectron LASer at Hamburg (FLASH) in combination with a synchronized femtosecond optical laser. Within a pump-probe scheme the IR pulse initiates a molecular fragmentation and then, after an adjustable time delay, the system is exposed to an intense FEL pulse. This way we follow the creation of highly-charged molecular fragments as a function of time, and probe the dynamics of multi-photon absorption during the transition from a molecule to individual atoms.

\section{Introduction}

How does intense XUV (extreme ultra-violet) or X-ray radiation couple with atoms, molecules, and matter in general? This question is in the focus of research with free-electron lasers (FELs) that provide femtosecond light pulses with unprecedented intensities of $10^{15} \mathrm{~W} \mathrm{~cm}^{-2}$ and more. Under these conditions atoms are ionized by multi-photon transitions, and charge states are reached that are inaccessible by single-photon absorption. ${ }^{\mathbf{1} 2}$ Moreover, atomic resonances were found to further enhance the efficiency of high charge-state production in heavy atoms. ${ }^{3,4}$ In contrast to the atomic case, experiments with rare-gas clusters yielded a more subtle picture. On the one hand, a large fraction of only singly charged

\footnotetext{
${ }^{a}$ Max-Planck-Institut für Kernphysik, 69117, Heidelberg, Germany

${ }^{b}$ Universität Kassel, 34132, Kassel, Germany

'J.R. MacDonald Laboratory, Department of Physics, Kansas State University, Manhattan, KS 66506, USA

${ }^{d}$ Max-Planck-Institut für medizinische Forschung, Heidelberg, Germany

${ }^{e}$ Max-Planck-Institut für Quantenoptik, 85748, Garching, Germany

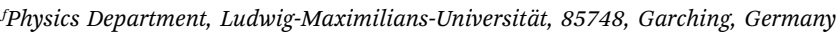

${ }^{8}$ Shanghai Advanced Research Institute, Chinese Academy of Sciences, 201210, Shanghai, China

${ }^{h}$ DESY, Notkestrasse 85, 22607, Hamburg, Germany

${ }^{i}$ Physikalisch-Technische Bundesanstalt, 38116, Braunschweig, Germany
} 
monomers were observed. ${ }^{5}$ The occurrence of these low charge states was assumed to be due to charge redistribution involving neighbouring neutral atoms as well as nano-plasma formation and recombination prior to fragmentation. On the other hand, intensity selective experiments in the soft X-ray regime revealed a complete absence of low charge states at highest intensities, a feature which is still under debate. ${ }^{6}$ The processes involved in radiation-induced fragmentation are, thus, strongly dependent not only on the atomic structure, the actual wavelength and the intensity, but also on the direct environment of a specific atom of interest embedded in a molecule, a cluster, or any complex system in general.

The understanding of the fragmentation dynamics of molecules irradiated by $\mathrm{X}$-ray photon pulses is crucial for the realization of single-molecule imaging with FELs which relies on the "diffract before destroy" concept. ${ }^{7}$ It must be ensured that the reconstructed structure is still that of the intact molecule. Therefore, the dynamics of absorption and charge redistribution as well as the accompanied nuclear motion is of interest on the femtosecond time scale, i.e. the typical duration of the FEL pulses. ${ }^{8}$ Particularly, the localized photon absorption at heavy constituents with large cross sections efficiently triggers electron rearrangement throughout the molecule within a few femtoseconds. For small molecules, this has been investigated in two recent studies on methylselenol ${ }^{9}$ and ethylselenol, ${ }^{\mathbf{1 0}}$ which contain selenium atoms as the heavy absorption centre. Even for 5 fs FEL pulses charge rearrangement and considerable displacement of the atomic constituents were observed.

Here, we trace the XUV absorption dynamics of iodine molecules $\left(\mathrm{I}_{2}\right)$ during the transition from a molecule to individual atoms. Besides the advantageous slow nuclear motion of the heavy iodine atoms the system exhibits strong similarities with Xe concerning photon-induced excitation and absorption cross sections in the XUV. Both, xenon and iodine are very efficiently excited with $\sim 90 \mathrm{eV}$ photons via shape or giant resonances which results in a highly effective coupling of intense FEL radiation. ${ }^{2}$ In the present experiment we use ultra-short XUV pulses of the Free-electron LASer at Hamburg (FLASH) in combination with a synchronized femtosecond optical laser. Within a pump-probe scheme the nuclear dynamics as well as the charge state of $I_{2}$ were followed as a function of time. The IR pulse initiates a molecular fragmentation and then, after an adjustable time delay, the system is exposed to an intense FEL pulse. By measuring the kinetic energies and the charge states of coincident ions that emerge from dissociating $\mathrm{I}_{2}$ molecules we monitor the inter-nuclear distance dependent absorption of intense FEL radiation. Our results confirm, and largely extend recent IR-XUV pump-probe studies with $\mathrm{I}_{2}$ at FLASH. ${ }^{11,12}$ In addition, changes in the ionization dynamics along the transition from molecular to atomic iodine are presented and discussed.

\section{Experimental setup}

The experiment was performed at FLASH using the focused beam $(\sim 30 \mu \mathrm{m}$ diameter) of beamline BL2. Pulses with $87 \mathrm{eV}$ photons were delivered at a repetition rate of $10 \mathrm{~Hz}$, and pulse durations of approximately 60 fs (FWHM) and intensities up to $3 \times 10^{14} \mathrm{~W} \mathrm{~cm}^{-2}$ were reached. The FEL beam was focused into a supersonic gas jet containing iodine molecules. Solid iodine was heated up to $400 \mathrm{~K}$ inside a reservoir containing helium gas at a stagnation pressure of about 
1 bar resulting in a $\mathrm{He}: \mathrm{I}_{2}$ ratio of approximately $50: 1$. The gas mixture was expanded through a heated $30 \mu \mathrm{m}$ diameter nozzle to create a supersonic jet. By means of a reaction microscope ${ }^{13}$ we detected in coincidence the ionic fragments that emerged from the ionization of target molecules by the FEL. While the XUV beam was directly focused into the gas jet, the IR laser was focused with a lens outside of the vacuum system, deflected by 90 degree with a holey mirror and overlapped collinearly with the FEL beam. Both foci were merged at the same point in the target (see Fig. 1). The IR laser provided $800 \mathrm{~nm}$ pulses synchronized with the FEL $(10 \mathrm{~Hz})$ with pulse durations of $\sim 80 \mathrm{fs}(\mathrm{FWHM}) \cdot{ }^{14}$ After focusing the IR beam onto a diameter of $50 \mu \mathrm{m}$ (FWHM) we reached a maximum intensity of approximately $3.4 \times 10^{14} \mathrm{~W} \mathrm{~cm}^{-2}$.

During the IR-XUV pump-probe experiments the arrival time of the IR laser was varied and continuously scanned over a range of $\sim 10$ ps in total. Unfortunately, due to technical reasons, some of the usually available IR-XUV synchronization tools for offline pulse arrival-time correction at FLASH were not running properly. Therefore, the pulse-to-pulse timing-jitter as well as long time drifts resulted in an overall temporal resolution for the arrival times between XUV and IR pulses on the order of one picosecond or slightly better. The spatial overlap was adjusted by simultaneously monitoring both beams on a screen that was moved into the interaction point. In order to visualize the XUV beam the screen is coated with a fluorescing powder (ZnS). The temporal overlap of the IR and XUV pulses was found by scanning the delay stage and monitoring the emission characteristic of the ions, which is different for a preceding or a delayed IR pulse.

Both beams, XUV and IR, were polarized along the gas-jet direction (i.e. in the drawing plane of Fig. 1). The ionic fragments created by ionization in the centre of the reaction microscope were accelerated perpendicular to the gas-jet beam and the FEL beam (i.e. out of the plane in Fig. 1) by a homogeneous electric field of

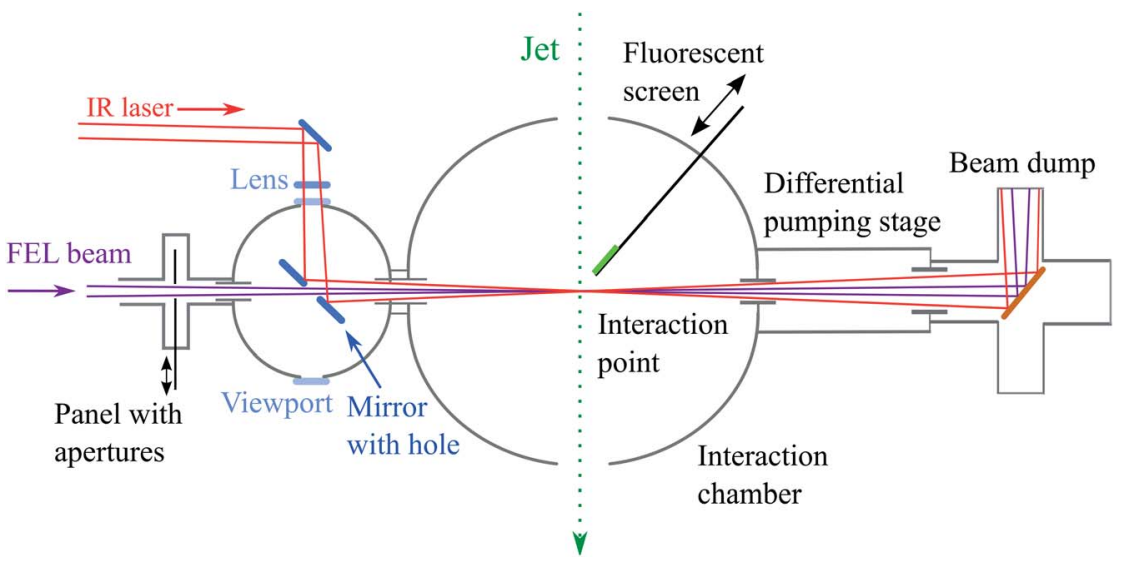

Fig. 1 Sketch of the experimental setup. The FEL beam passes the IR deflection mirror from behind through a $5 \mathrm{~mm}$ hole. The XUV and IR beam are focused into the gas target and dumped onto a tilted copper blade to suppress stray light. Differential pumping stages before and behind the interaction chamber ensure XHV conditions $\left(<10^{-11} \mathrm{mbar}\right)$ at the interaction point. The main chamber comprises a reaction microscope (not shown, see text) for coincident ion detection. 
$35 \mathrm{~V} \mathrm{~cm}^{-1}$ onto a large area channel-plate detector $(120 \mathrm{~mm}$ diameter) with position readout $(0.1 \mathrm{~mm}$ resolution). The time-of-flight (TOF) and position information allows us to reconstruct the three-dimensional momentum of each ion. As most of them emerge from the Coulomb explosion of multiply charged $\mathrm{I}_{2}$ molecules they carry a significant amount of kinetic energy (up to $100 \mathrm{eV}$ ). This complicates the unique assignment of ion pairs $\mathrm{I}^{\mathrm{Q1+}}$ and $\mathrm{I}^{\mathrm{Q2+}}$ that emerge from one molecule with a total charge of $Q 1+Q 2$ because the flight-time distributions for different species start to overlap. The charge state separation by the ions' timeof-flight alone is hardly possible for such high kinetic energies. Therefore, we use the method of coincident ion detection and the application of momentum conservation to unambiguously identify the two ions that belong to one molecule. In the analysis we request that the vector sum of the momenta of two iodine ions is equal to zero (within the experimental resolution). In practice this means that for a detected particle A we search the list of additionally registered ions for a particle B such that their momentum vectors cancel each other. This is done for every FEL pulse. In order to calculate the momentum vectors we need to assume a charge $Q 1$ for ion A and a charge $Q 2$ for ion B. If we guess wrong the momentum conservation is likely to fail in at least one spatial coordinate. In this way we find the charge combinations of coincident detected pairs of ions, even if more than one molecule has been ionized by the laser. The confidence that the correct ion pairs are assigned is estimated to be $98 \%$ for $\mathrm{I}^{1+}+\mathrm{I}^{1+}$ and $80 \%$ for $\mathrm{I}^{7+}+\mathrm{I}^{8+}$.

\section{Ionization of $I_{2}$ with intense XUV radiation}

Ionization of iodine at a photon energy of $87 \mathrm{eV}$ proceeds predominantly by electron emission from the $4 \mathrm{~d}$ shell, ${ }^{15}$ which exhibits a large cross section (more than $10 \mathrm{Mb}$ ), in close analogy to the $4 \mathrm{~d}$ giant resonance in Xe. To understand the pathways leading to the observed charge states in $\mathrm{I}_{2}$ we first will briefly discuss the ionization of atomic iodine. The absorption of one photon creates a $4 \mathrm{~d}$ inner-shell vacancy that relaxes by one or two Auger processes ${ }^{16}$ resulting in $\mathrm{I}^{2+}$ or $\mathrm{I}^{3+}$, respectively. The cross section for ionization of $\mathrm{I}^{2+}$ is also dominated by the $4 \mathrm{~d}$ absorption, which initiates another Auger process leading to $\mathrm{I}^{4+} \cdot{ }^{17}$ The typical Auger decay times on the order of 20 fs are shorter than the FEL pulse duration used here. For charge states $\mathrm{I}^{3+}$ and higher, $5 \mathrm{p}$ valence-shell ionization becomes dominant increasing the charge state by only one per absorbed photon. The $5 \mathrm{p}$ shell is completely depleted by the time $\mathrm{I}^{5+}$ is reached and thus the $5 \mathrm{~s}$ shell will be emptied. The energy needed for the transition from $\mathrm{I}^{6+}$ to $\mathrm{I}^{7+}$ is at the limit of the photon energy of $87 \mathrm{eV}$ (see Table 1), but may still be accessible due to the broad bandwidth of the FEL. For the transition from $\mathrm{I}^{7+}$ to $\mathrm{I}^{8+}$ two photons are required to overcome the ionization threshold, and finally, for the step from $\mathrm{I}^{9+}$ to $\mathrm{I}^{10+}$ even three photons are needed.

For the discussion of XUV ionization of molecular $\mathrm{I}_{2}$ we again need the ionization potentials up to the highest observed charge states $\mathrm{I}_{2}{ }^{16+}$. As the experimentally known valence ionization potential only ranges up to $\mathrm{I}_{2}{ }^{2+},{ }^{19}$ we use a simple model to estimate the missing values. This is done by approximating the ionization potential of just the least bound electron as this is the most relevant one for the production of the high charge states. Molecular properties are neglected in the following. For an iodine ion $\mathrm{I}^{Q^{+}}$, the influence of a neighbouring ion $\mathrm{I}^{P+}$ (with $P>Q$ ) is taken into account by assuming that the Coulomb potential 
Table 1 The lowest ionization potentials $\mathrm{E}_{Q}$ for atomic iodine $\mathrm{I}^{Q+}$ and estimated ionization energies $E_{Q}^{\prime}$ for the molecule $\mathrm{I}_{2}{ }^{Q+}$. The latter are calculated for a fixed inter-nuclear distance of $R_{\mathrm{eq}}=5.0$ a.u., i.e. that of the neutral molecule (see text). The atomic ionization potentials are taken from ref. 18. Additionally listed are the minimum numbers of photons $(87 \mathrm{eV})$ needed for the indicated ionization step

\begin{tabular}{|c|c|c|c|c|}
\hline$Q^{+} \rightarrow(Q+1)+$ & $\begin{array}{l}E_{Q}(\mathrm{eV}) \\
\mathrm{I}^{Q^{+}} \rightarrow \mathrm{I}^{(Q+1)+}\end{array}$ & Photon Number & $\begin{array}{l}E_{Q}^{\prime} \\
(\mathrm{eV}) \mathrm{I}_{2}{ }^{Q+} \rightarrow \mathrm{I}_{2}^{(Q+1)+}\end{array}$ & Photon Number \\
\hline $0 \rightarrow 1$ & 10.4 & 1 & 9.35 & 1 \\
\hline $1 \rightarrow 2$ & 19.1 & 1 & 15.7 & 1 \\
\hline $2 \rightarrow 3$ & 29.6 & 1 & 24.4 & 1 \\
\hline $3 \rightarrow 4$ & 40.4 & 1 & 29.7 & 1 \\
\hline $4 \rightarrow 5$ & 51.2 & 1 & 40.2 & 1 \\
\hline $5 \rightarrow 6$ & 74.4 & 1 & 45.5 & 1 \\
\hline $6 \rightarrow 7$ & 87.6 & 1 & 56.3 & 1 \\
\hline $7 \rightarrow 8$ & 150.8 & 2 & 61.6 & 1 \\
\hline $8 \rightarrow 9$ & 171.0 & 2 & 72.4 & 1 \\
\hline $9 \rightarrow 10$ & 197.0 & 3 & 77.7 & 1 \\
\hline $10 \rightarrow 11$ & - & - & 100.9 & 2 \\
\hline $11 \rightarrow 12$ & - & - & 106.2 & 2 \\
\hline $12 \rightarrow 13$ & - & - & 119.4 & 2 \\
\hline $13 \rightarrow 14$ & - & - & 124.7 & 2 \\
\hline $14 \rightarrow 15$ & - & - & 187.9 & 3 \\
\hline $15 \rightarrow 16$ & - & - & 193.2 & 3 \\
\hline $16 \rightarrow 17$ & - & - & 213.4 & 3 \\
\hline $17 \rightarrow 18$ & - & - & 213.4 & 3 \\
\hline
\end{tabular}

of $\mathrm{I}^{P+}$ leads to tighter bound electrons of $\mathrm{I}^{Q+}$. Thus, the binding energy of the least bound electron of $\mathrm{I}^{Q^{+}}\left(\mathrm{E}_{Q}<0\right)$ is increased to $\mathrm{E}_{Q}^{\prime}=\mathrm{E}_{Q}-P / R$, where $R$ is the internuclear distance between the two ions (atomic units, a.u., are used throughout

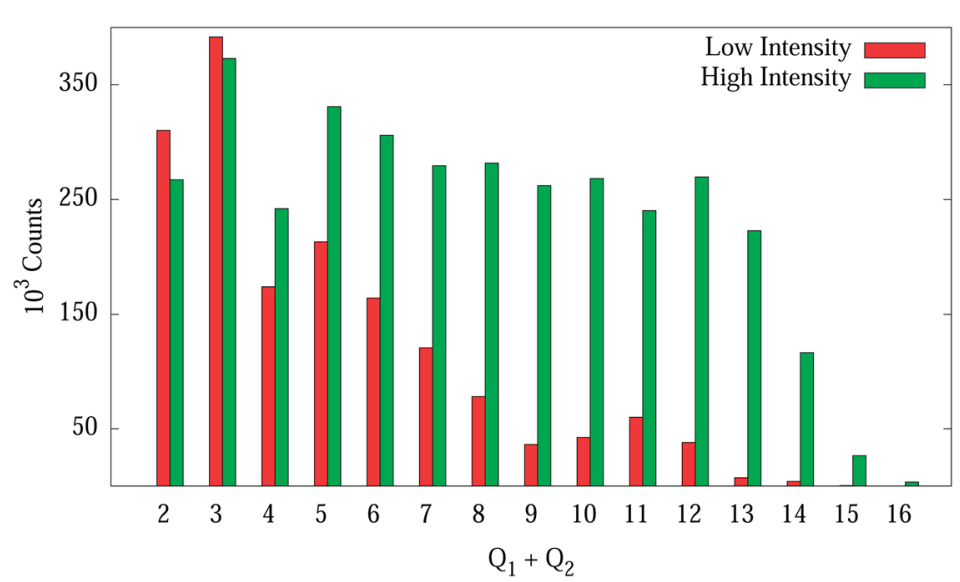

Fig. 2 Measured charge state distributions for multiple ionization of molecular iodine $\mathrm{I}_{2}^{(Q 1+Q 2)+}$ at a low FEL intensity $\left(\sim 1 \times 10^{14} \mathrm{~W} \mathrm{~cm}^{-2}\right.$, red bars $)$ and high intensity $\left(\sim 3 \times 10^{14}\right.$ $\mathrm{W} \mathrm{cm} \mathrm{cm}^{-2}$, green bars). The data are taken with XUV pulses only. The total charge state of the molecule is inferred from the charges of coincident ion pairs $1^{Q 1+}+1^{Q 2+}$. Counts of ion pairs are plotted as a function of their sum charges. The small amount of low charge states in the high-intensity measurement may be attributed to saturation effects in the ion detection system. 
with the electron charge, its mass and the Planck constant $e=m_{\mathrm{e}}=\hbar=1$ ). The molecule is assumed to fragment into the most symmetric configuration, e.g. $\mathrm{I}_{2}{ }^{5+}$ dissociates into $\mathrm{I}^{2+}+\mathrm{I}^{3+}$. The estimated ionization potentials for the transition $\mathrm{I}_{2}{ }^{Q^{+}} \rightarrow \mathrm{I}_{2}{ }^{(Q+1)+}$ at the equilibrium inter-nuclear distance $R_{\mathrm{eq}}=5.0$ a.u. are listed in Table 1 . To test the quality of our estimate we compare the experimentally determined double-ionization potential $\mathrm{E}\left(\mathrm{I}_{2} \rightarrow \mathrm{I}_{2}{ }^{2+}\right)=(24.95 \pm 0.02) \mathrm{eV}^{19}$ with our calculated value of $25.05 \mathrm{eV}$ from Table 1 . Both are in very good agreement, supporting our conclusions.

We will now turn to the ionization pathways in molecular $\mathrm{I}_{2}$. In analogy to atomic iodine a single photon creates $\mathrm{I}_{2}{ }^{2+}$ or $\mathrm{I}_{2}{ }^{3+}$ as a result of $4 \mathrm{~d}$ ionization and subsequent Auger emission. We assume that a second photon interacting with $\mathrm{I}_{2}{ }^{2+}$ and $\mathrm{I}_{2}{ }^{3+}$ mainly creates further $4 \mathrm{~d}$ vacancies which again relax via Auger decay ${ }^{19}$ leading to $\mathrm{I}_{2}{ }^{4+}$ and $\mathrm{I}_{2}{ }^{5+}$, respectively.

From $\mathrm{I}_{2}{ }^{4+}$ onwards $4 \mathrm{~d}$ inner-shell ionization is competing with valence ionization. This we conclude from the measured charge state ratios of $\mathrm{I}_{2}{ }^{4+}$ and $\mathrm{I}_{2}{ }^{5+}$ ions in Fig. 2, where the distributions of total molecular charge states for two different FEL intensities are shown. The yield of $\mathrm{I}_{2}{ }^{5+}$ ions is enhanced because it features two precursors. However, the exact ratio between inner-shell and valence ionization is not known. Finally, for charge states $\mathrm{I}_{2}{ }^{6+}$ and higher only single valence ionization is energetically allowed, and the charge state increases by one per photon. The highest possible charge state that can be reached by sequential single-photon absorption events is $\mathrm{I}_{2}{ }^{10+}$ according to our estimated ionization potentials in Table 1 . If one considers ionization of two individual atoms a

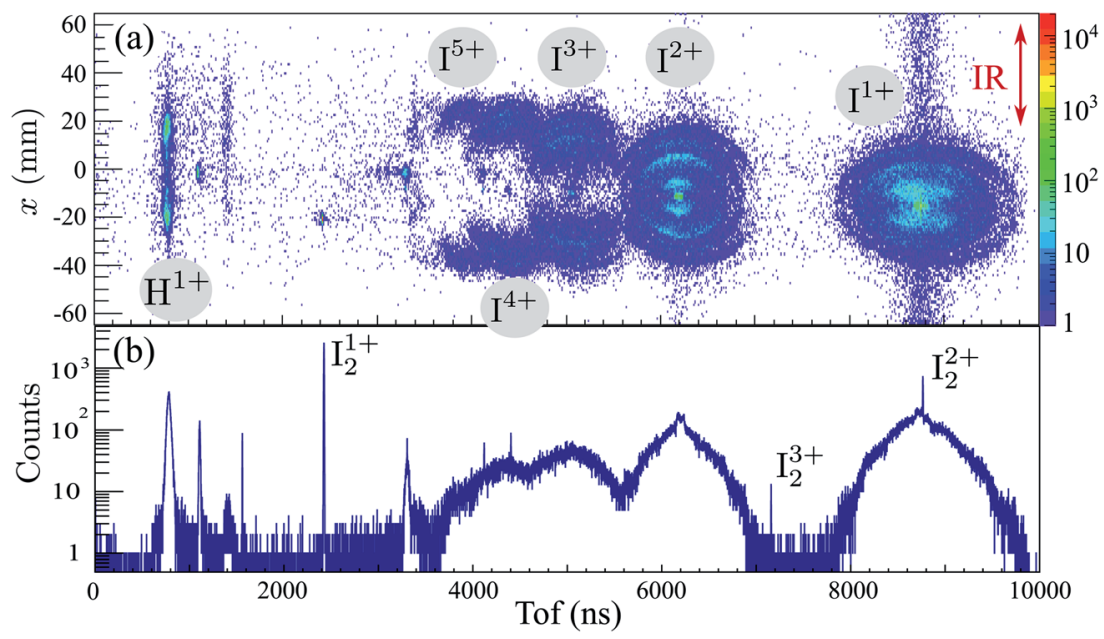

Fig. 3 Ionization of $\mathrm{I}_{2}$ by IR laser pulses only. (a): Ion time-of-flight (TOF) versus $x$ coordinate of the hit position on the detector (the direction along the laser polarization). Dominant fragments originating from Coulomb explosion of multiply charged $\mathrm{I}_{2}$ are indicated as well as protons emerging from residual-gas $\left(\mathrm{H}_{2}\right)$ ionization. As the gas jet is propagating into the negative $x$-direction all fragments emerging from the moving target show a constant $x$-offset. (b): Projection of (a) onto the TOF axis. Note that due to technical reasons flight times are sorted modulo a maximum time of $10 \mu \mathrm{s}$, meaning that the $\mathrm{I}_{2}{ }^{1+}$ peak does not appear at the expected TOF $=12400 \mathrm{~ns}$ but at the beginning of the spectrum (at TOF-10000 ns). 
maximum charge state of $\mathrm{I}_{2}{ }^{14+}$ is expected. From then on direct two-photon absorption is required for further ionization. In the experiment we see a clear falloff in the yield above about $\mathrm{I}_{2}{ }^{12+}$ (Fig. 2). To reach this charge state $(Q 1+Q 2=12)$ the stepwise absorption of at least 8 photons is required. At first, this may seem in contradiction to our estimated molecular ionization potentials listed in Table 1. There, direct two-photon absorption is required from $\mathrm{I}_{2}{ }^{10+}$ onwards. However, it should be recalled that these binding energies are estimated for a fixed internuclear distance of $R_{\mathrm{eq}}=5.0 \mathrm{a}$.u. This assumption is not valid because ionization often triggers a dissociation of the molecule while more photons get absorbed within the pulse duration. With increasing inter-nuclear distance the ionization potential is lowered. Hence, during the course of sequential photon absorption the electronic binding energies are expected to approach the values of individual atoms or ions. Reaching higher charge states than expected may also be possible due to resonantly excited intermediate states. While a direct ionization with one photon is energetically not allowed from a certain charge state on, it might well be possible for a previously excited ion. If the excited ion is created by a resonant transition with large cross sections, ionization might still be very efficient even though the required energy is above the single-photon ionization threshold. This effect of resonance enhanced ionization was observed for $\mathrm{Xe}$ and $\mathrm{Kr}$ at higher photon energies.,

All considerations above are made under the assumption that Auger decay has already occurred before the next photoionization takes place. The situation is different if the first $4 \mathrm{~d}$ photoionization is followed by a second or third one before Auger decay has happened. This way so-called hollow atoms with multiple core holes are created, where the inner-shells are depleted. ${ }^{20,21}$ In general this requires higher intensities, shorter pulses, or larger photon energies where longer, more complicated Auger cascades are initiated. Therefore, we assume that these effects are negligible for our FEL pulse parameters.

\section{Ionization of $\mathrm{I}_{2}$ in strong IR laser fields}

While the XUV photons mainly interact with inner-shell electrons, the IR laser field couples most effectively to the least bound electrons in the outer shells. The field imposes a strong perturbation on the molecule that may lead to multiphoton or tunnel ionization. The ionization dynamics of diatomic homo-nuclear molecules in intense femtosecond IR laser pulses has been investigated intensively ${ }^{22-24}$ including $\mathrm{I}_{2}$ due to its comparably slow dissociation dynamics..$^{22,25,26}$

Once a molecule is multiply ionized it will most likely dissociate while it may absorb more IR photons that further ionize it. This accelerates the fragments because the Coulomb repulsion increases with the charge. In the literature this process is referred to as multi-electron dissociative ionization (MEDI). ${ }^{27}$ Analyzing the fragments' kinetic energies gives insight into the ionization process on a time scale that is comparable to the dissociation time. The MEDI scheme occurs only for some molecules at certain wavelengths but fails for others. ${ }^{28,29}$ An alternative scheme, where the ionization probability was found to be drastically enhanced at certain inter-nuclear distances, ${ }^{\mathbf{3 0}}$ is the so-called charge-resonance-enhanced ionization (CREI). ${ }^{24,31}$ The distance for enhanced ionization of $\mathrm{I}_{2}$ is approximately two times the equilibrium inter-nuclear distance. ${ }^{22}$ 
An overview of ion fragments measured only with the IR laser is shown in Fig. 3. The data are recorded at a repetition rate of $10 \mathrm{~Hz}$, the maximum repetition rate of the IR laser system, and an intensity of about $3.4 \times 10^{14} \mathrm{~W} \mathrm{~cm}^{-2}$ (the estimated pulse length was $80 \mathrm{fs}$ ). In Fig. 3a the ion times-of-flight (TOF) are plotted against their impact position at the detector. Particles with zero initial momentum appear at the origin of position $(\mathrm{x}=0)$ and at a sharp TOF defined by the charge-to-mass ratio. Ions with a non-zero momentum along the target-pointdetector axis exhibit a change in the TOF, and those with a momentum into the gas-jet or polarization direction are displaced along the $x$-position of detection. Thus, Fig. 3a represents an image of the combined ion charge and momentum distribution.

The molecules in the gas target are randomly oriented, but those with their inter-nuclear axis parallel to the laser polarization are ionized most efficiently by the IR laser, resulting in a predominant ion emission along this axis. This is clearly seen in the data as dipole-like ion distributions. Assuming axial recoiling, the original orientation can be extracted from the measured fragment emission angles after Coulomb explosion of the molecule. As the IR laser couples very efficiently to the least bound valence electrons those from the $5 \mathrm{p}$-like shells are ionized first. A strong indicator for this hypothesis is the charge-state distribution showing a sharp cut-off beyond $\mathrm{I}^{5+}$, which corresponds to a completely depleted $5 \mathrm{p}$ shell in atomic iodine. The present intensity is sufficient to create fragments up to $\mathrm{I}^{5+}$ in this transition regime between multi-photon absorption and tunnel ionization.

The distribution of each charge state in Fig. 3(a) shows several discrete ringlike structures, with larger radii for higher charge states. The larger the radius, the higher the energy gained from Coulomb explosion. For instance, the distribution of $\mathrm{I}^{2+}$ ions features four rings, each from a Coulomb explosion with one fragment being $\mathrm{I}^{2+}$. As the IR laser mostly creates dissociating
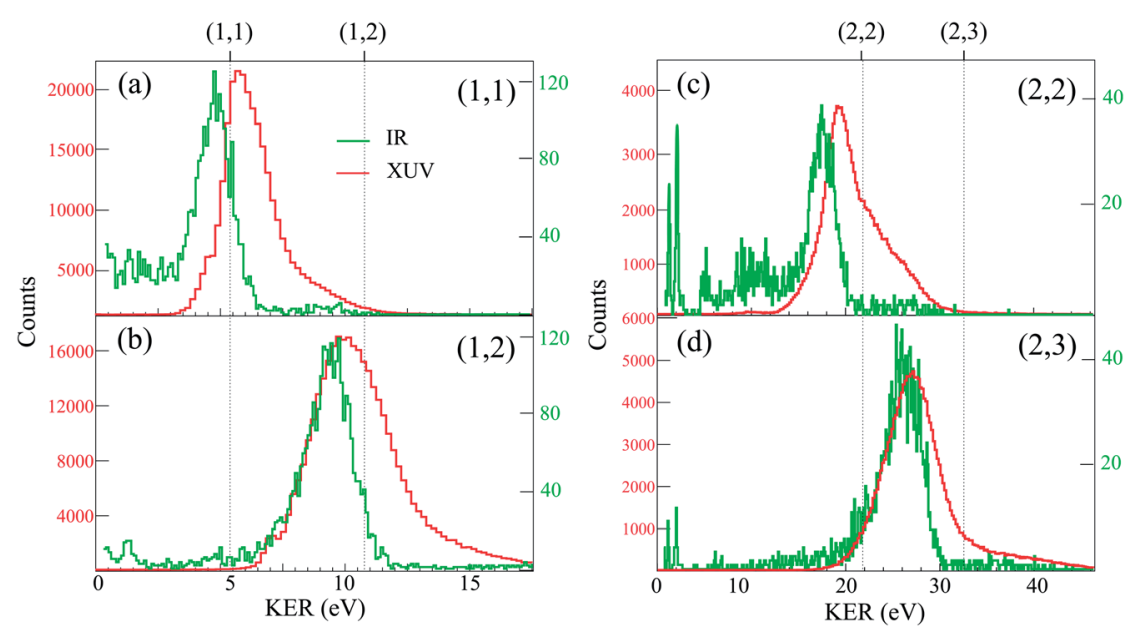

Fig. 4 Comparisons of kinetic energy release (KER) spectra obtained with single XUV (red) and single IR pulses (green). The final charges of coincident ions $\left.\right|^{Q 1+}+\left.\right|^{Q 2+}$ are indicated in brackets (Q1, Q2). The XUV pulses were roughly 60 fs long and the intensity was $\sim 1 \times 10^{14}$ $\mathrm{W} \mathrm{cm}{ }^{-2}$. The estimated IR intensity is about $3 \times 10^{14} \mathrm{~W} \mathrm{~cm}^{-2}$. 
molecules with at least one neutral fragment, ${ }^{12}$ the lowest energy and thus the innermost ring corresponds to the fragmentation channel $\mathrm{I}^{2+}+\mathrm{I}^{0}$, the next outer one to $\mathrm{I}^{2+}+\mathrm{I}^{1+}$, and so forth.

For fragments with charge states up to $\mathrm{I}^{4+}$ a maximum in the centre of each Coulomb-explosion ring (see Fig. 3) is visible, which is due to fragments with very low kinetic energies. This is only possible for fragmentation into a multiply charged ion and a neutral atom. At first glance this might be surprising, because the symmetric channels are energetically more favourable. However, for short pulses and at high intensities the IR field bends the molecular potential such that tunnelling ionization leads to asymmetrically charged fragments. ${ }^{22,26}$ In addition, we cannot exclude that these events are partly due to a weak pre-pulse or pedestal of the IR pulse that leads to dissociation before the main IR pulse impinges. Energetically, only two $1.55 \mathrm{eV}$ photons are needed to dissociate the neutral $\mathrm{I}_{2}$ molecule.

Distributions of total ion kinetic energies (KER) obtained with single IR and single XUV pulses are shown in Fig. 4 for various final charge-state combinations. In the following, coincident ion pairs will be denoted as $(Q 1, Q 2)$ instead of $\mathrm{I}^{Q 1+}+\mathrm{I}^{Q 2+}$. For the IR data, the KER spectra of the fragmentation channels $(1,1)$ and $(2,2)$ are clearly shifted towards lower values compared to the XUV case. This indicates that also in the IR pulse the charging-up occurs stepwise over a time period that is comparable to the pulse length ( $80 \mathrm{fs}$ ). The molecule is already in the progress of dissociation at the moment when the final charge state is reached resulting in a less energetic Coulomb explosion and, thus, a smaller KER. In fact, several IR photons are needed to reach $(1,1)$, while a single XUV photon is sufficient. Because the $(1,1)$ channel is a precursor of $(2,2)$ a similar trend for the KER peak is expected for the $(2,2)$ channel. In principle, the same argumentation holds for the channels $(1,2)$ and $(2,3)$. However, here the IR and XUV spectra share their low-energetic cut-off, indicating a slightly delayed population of the final charge states for the XUV pulses as well. This can be explained again with a sequential, stepwise absorption of two XUV photons during the FEL pulse. Some of the XUV created KER spectra are significantly broader than those for the IR (Fig. $4 \mathrm{~b}$ and $4 \mathrm{c}$ ). At the present stage we can only speculate about the possible reason. It might be that the time-delayed emission of Auger electrons after XUV inner valence ionization causes this broadening. While the $(1,1)$ state is produced after the first Auger decay, the second Auger emission leads to the creation of $(1,2)$. The exponential nature of the delayed Auger emission may cause a broadening in the case that dissociation has already started before the second Auger decay happens. If the decay occurs very fast we expect a high KER, if it lives longer the KER is small.

\section{IR-XUV pump-probe results}

Having introduced the ionization mechanisms in single XUV and single IR pulses we now turn to the discussion of the IR-XUV pump-probe results. The intensity of the IR pulse is identical to that used for the measurement discussed in the previous chapter, and the intensity of the XUV pulses is roughly $3 \times 10^{14} \mathrm{~W} \mathrm{~cm}^{-2}$. The already introduced spectra of ion TOF versus hit position on the detector are presented in Fig. 5 for the two cases of IR-early (top) and IR-late (bottom). 


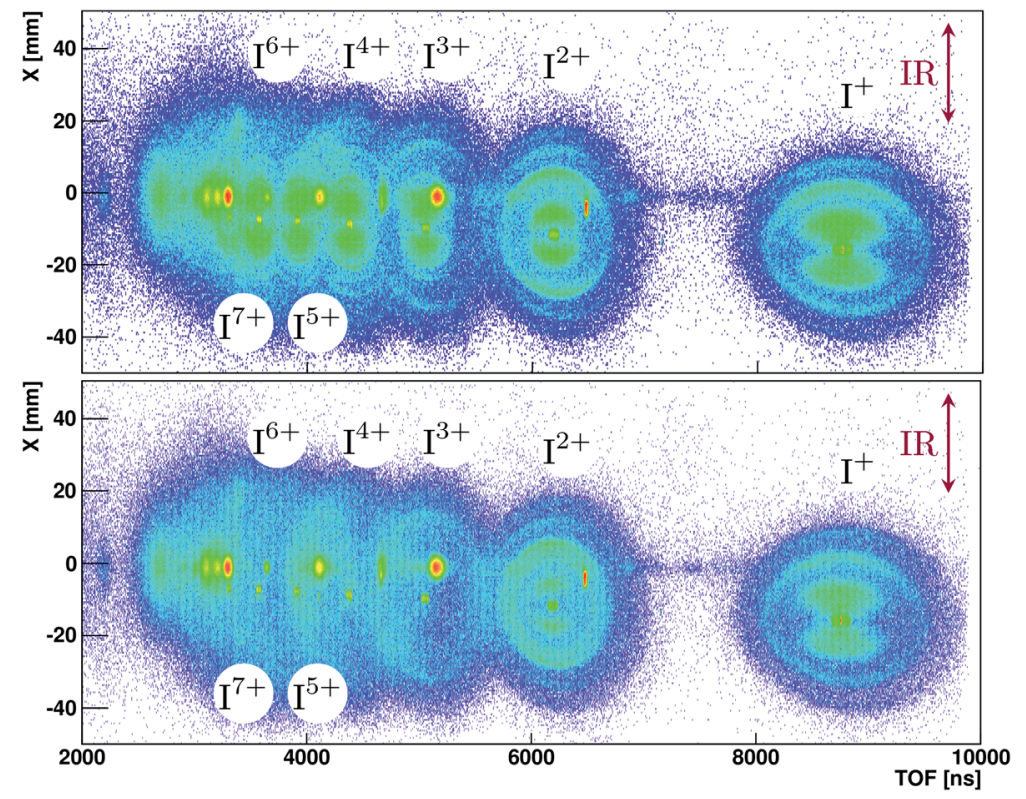

Fig. 5 TOF versus the $x$-position on the detector for (a) when the IR pulse arrives before the XUV pulse and (b) the IR is later than the XUV pulse.

If the IR pulse arrives first it efficiently initiates the dissociation of the molecule. Thus, the laser polarisation determines the emission direction of the coincident fragments. Therefore, the dipole-shaped emission pattern along the polarization axis is preserved even after interaction with the XUV pulse. This is true for all charge states including those that are out of reach with the IR laser only as shown in Fig. 5a. If the XUV pulse arrives first, the emission characteristic is almost spherically symmetric. This is expected because the XUV ionization cross-section is roughly independent of the molecular alignment. For small charge states the emission characteristic remains partially dipole-like because some of those events are created with the IR pulse alone.

The delay-dependent KER spectra for symmetric or almost symmetric coincidence channels are shown in Fig. 6. All time-independent, horizontal features are created within a single pulse, either XUV or IR, and the time-dependent traces stem from the joint interaction of both pulses, XUV and IR. They show a decreasing KER as the delay gets larger, finally converging to an asymptotic value. This is due to the creation of intermediate charge states $\left(Q 1_{\mathrm{i}}, Q 2_{\mathrm{i}}\right)$ along with dissociation of the molecule after the pump pulse. Both ions move apart until the probe pulse further ionizes the system promoting it onto a steeper Coulomb curve belonging to the final charge states $\left(Q 1_{\mathrm{f}}, Q 2_{\mathrm{f}}\right)$. The longer the delay the smaller the energy gain on the steeper potential energy curve gets. For very large delays the measured KER is equal to that of the intermediate or precursor state $\left(Q 1_{i}, Q 2_{i}\right)$. Thus, the time-dependent traces allow us to reconstruct the full reaction pathway from the neutral molecule to the intermediate state after the pump pulse $\left(Q 1_{\mathrm{i}}\right.$, $Q 2_{\mathrm{i}}$ ), as well as from this precursor state to the final Coulomb explosion channel $\left(Q 1_{\mathrm{f}}, Q 2_{\mathrm{f}}\right)$ after the probe pulse. Also shown in Fig. 6 are expected KER values for 

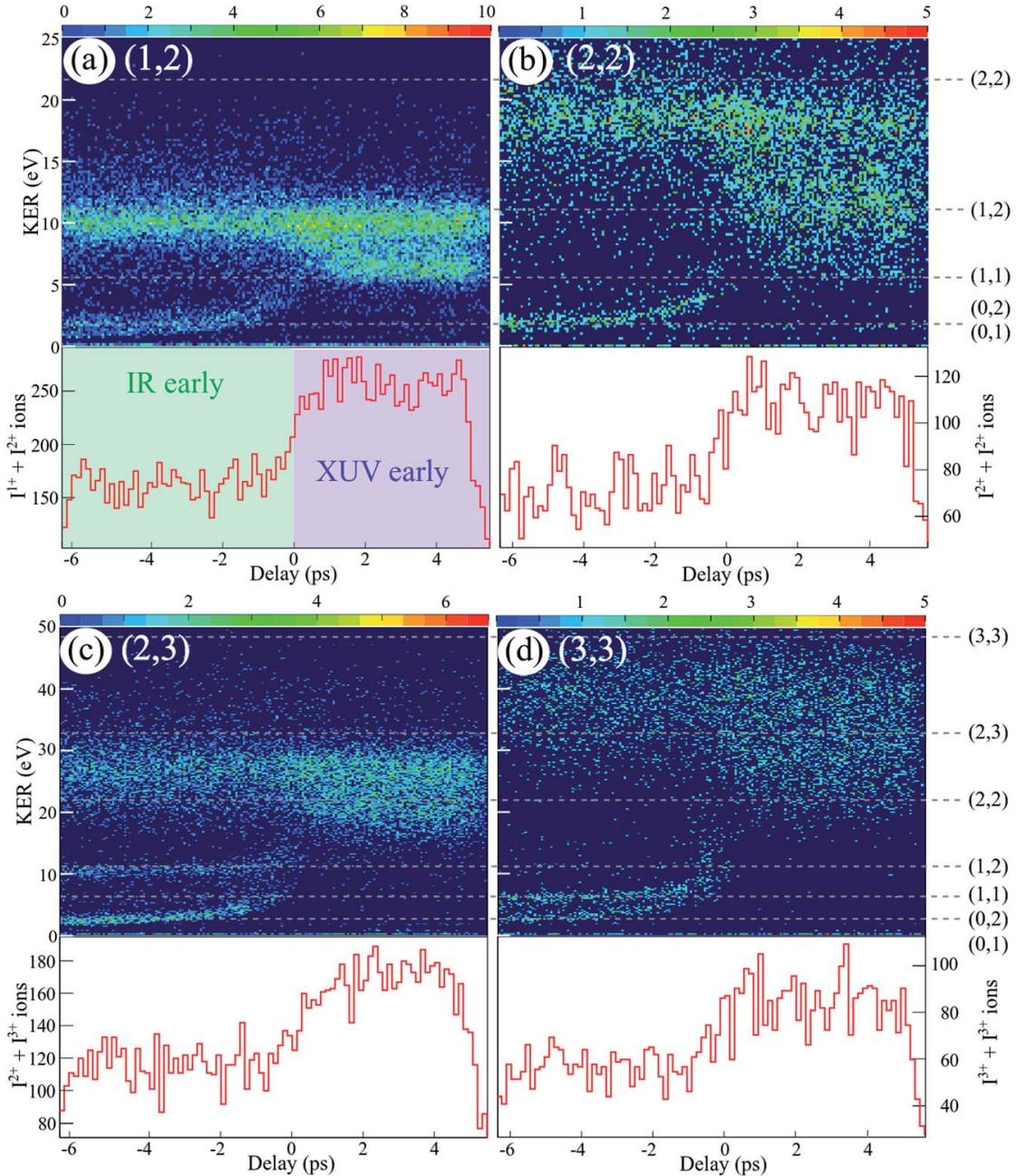

Fig. 6 Delay-dependent KER spectra for the (more) symmetric coincidence channels as indicated along with their projection onto the delay axis. Negative delays denote the case of a preceding IR pulse and positive values stand for a delayed IR pulse. Expected kinetic energy releases calculated with the Coulomb-explosion model are superimposed (grey dashed lines) for channels shown on the right side in order to identify the corresponding precursor states of each coincidence channel.

various fragmentation channels. These values are calculated assuming purely Coulombic potential energy curves $\mathrm{KER}=\mathrm{E}_{\mathrm{Coul}}=(Q 1 \cdot Q 2) / R$ (in atomic units). For example, for the instantaneous ionization $I_{2} \rightarrow(1,2)$ at the equilibrium internuclear distance $R_{\text {eq }}=5.0$ a.u. the expected KER is $11 \mathrm{eV}$.

We now discuss the case of negative delays where the IR pulse is preceding. From Fig. 6 we conclude that for the coincidence channels $(1,2)$ and $(2,2)$ only low-energy precursors with one neutral fragment are involved. As a delayed XUV pulse will always increase the charge state of one of the fragments by two, the most likely pathway for the production of channel $(1,2)$ is via $(1,0)$ and for $(2,2)$ via $(2,0)$ : 


$$
\mathrm{I}_{2} \stackrel{I R}{\longrightarrow}(1,0) \stackrel{X U V}{\longrightarrow}(1,2) \mathrm{I}_{2} \stackrel{I R}{\longrightarrow}(2,0) \stackrel{X U V}{\longrightarrow}(2,2)
$$

The higher final charge states, $(2,3)$ and $(3,3)$, feature neutral and charged precursor states as indicated in Fig. $6 \mathrm{c}$ and $6 \mathrm{~d}$, respectively. The precursors with a neutral fragment are assigned as above. The charged precursors are identified using the Coulomb explosion model to be $(1,2)$ and $(1,1)$, respectively. Thus the spectra with preceding IR laser can be summarized in the following way:

$$
\begin{aligned}
& \mathrm{I}_{2} \stackrel{I R}{\longrightarrow}(2,1) \stackrel{X U V}{\longrightarrow}(2,3) \mathrm{I}_{2} \stackrel{I R}{\longrightarrow}(2,0) \stackrel{X U V}{\longrightarrow}(2,3) \\
& \mathrm{I}_{2} \stackrel{I R}{\longrightarrow}(1,1) \stackrel{X U V}{\longrightarrow}(3,3) \mathrm{I}_{2} \stackrel{I R}{\longrightarrow}(1,0) \stackrel{X U V}{\longrightarrow}(3,3)
\end{aligned}
$$

For a preceding XUV pulse the spectra are analyzed in exactly the same way, but neutral precursors can be neglected. The delay-dependent part of the final channel $(1,2)$ for instance is reached by producing the precursor $(1,1)$ and subsequent removal of a further electron by the IR probe. Similarly, $(2,2)$ is created via the main precursor $(1,2)$ with a weak contribution from the precursor $(1,1)$. Interestingly the channel $(2,3)$ does only show a single precursor, $(2,2)$, while the next higher coincidence channel $(3,3)$ again features two precursors. The question arises why $(1,2)$, the second possible precursor of $(2,3)$, is missing.

Interestingly, in several cases the IR laser effectively ionizes already singly or doubly charged fragments created by XUV absorption, as can be seen e.g. in Fig. $6 \mathrm{~b}$ and $6 \mathrm{c}$, where a $(1,1)$ precursor contributes to the channel $(2,2)$, or the intermediate $(2,2)$ state leads to $(3,3)$ after the IR pulse (Fig. $6 \mathrm{~d})$. The XUV innervalence ionized molecular ion or, correspondingly, the ionic fragments may remain in a highly excited state which are easily further ionized by the IR pulse. The results demonstrate that delayed ionization by the IR laser is a very efficient

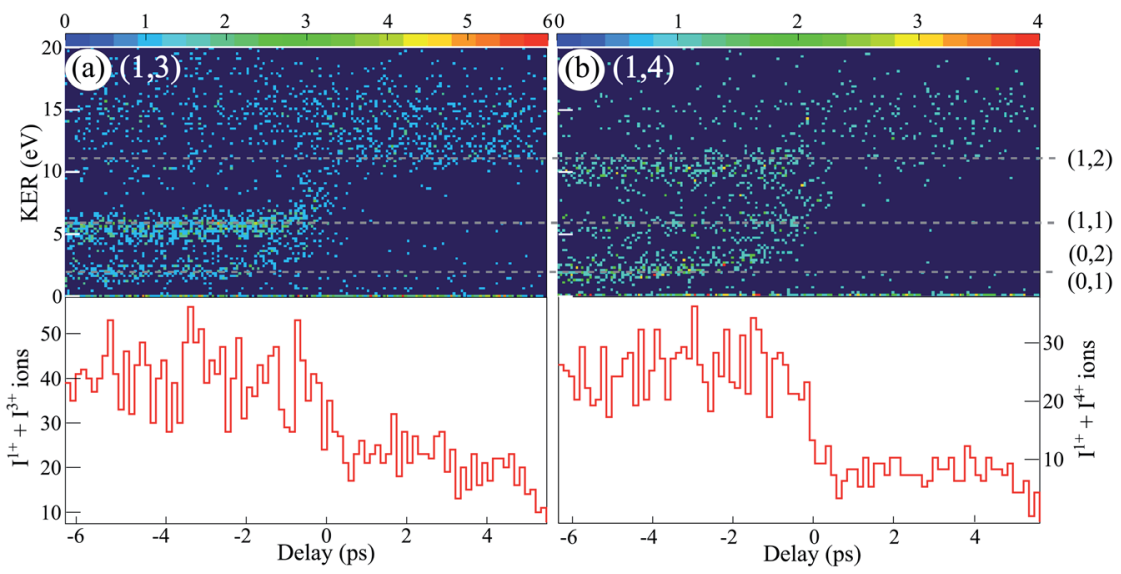

Fig. 7 Delay-dependent KER spectra for the asymmetric coincidence channels $(1,3)$ and $(1,4)$ and their projections onto the delay axis. Possible precursor charge states are indicated as dashed lines. 
process, because it clearly dominates the ion yield of the delay-dependent KER spectra in Fig. 6. However, this trend of an increased ion yield at positive delays is only present for the more symmetric break-up channels and only up to a certain degree of ionization. For already highly charged molecular ions the IR laser intensity is not sufficient anymore to strip off further electrons, in particular if all electrons are removed from the $5 \mathrm{p}$ shell (beyond $\mathrm{I}^{5+}$ ).

As mentioned, the asymmetric channels exhibit an opposite behaviour with a decrease of the ion yield for positive delays (XUV early). This is shown in Fig. 7, where the delay-dependent KER spectra are plotted for the coincidence channels $(1,3)$ and $(1,4)$. From this we draw the conclusion that delayed ionization by the IR laser favours the production of symmetric charge-state distributions. An IR pulse, which couples best to outer-shell electrons, will thus not create asymmetric charge states, except for very strong fields, when the molecular potential is bent so strongly that the asymmetric break-up gets energetically more favourable than the symmetric. ${ }^{22}$ Furthermore, even for large positive delays, where the iodine ions are considered as separated, the IR laser does not create asymmetric charge states. If for instance the XUV pulse ionizes $I_{2}$ into $(1,2)$ and the separated ionic fragments are placed into the IR field the number of photons needed to remove another electron from $\mathrm{I}^{+}$is much smaller than that for $\mathrm{I}^{2+}$. Therefore the creation of $(1,3)$ compared to $(2,2)$ is suppressed. This explanation is valid for the creation of all asymmetric charge states. By contrast, if the XUV pulse arrives late, a single photon is sufficient to even doubly ionize either of the ions and thus asymmetric charge states are created.

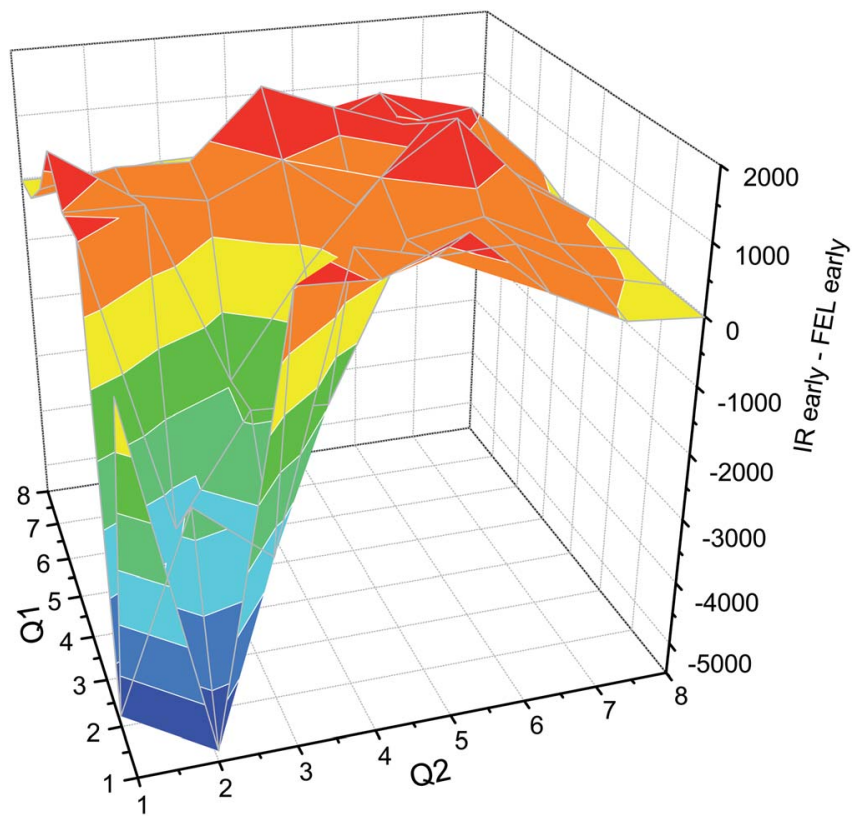

Fig. 8 Yield difference (in arbitrary units) between the two situations of a preceding IR and a successive IR pulse (with respect to the XUV pulse) as a function of Q1 and Q2. A value larger than zero means that the corresponding channel $(Q 1, Q 2)$ exhibits a larger yield if the IR comes first, and vice versa. For better visibility the distribution is symmetrized with respect to the diagonal $Q 1=Q 2$. 


\section{Summary and conclusions}

We conclude with an overview of our results and by providing an answer to the question whether higher charge states are reached for ionization of the bound molecule or two separated atoms. The role of the preceding IR pulse is to prepare two isolated neutrals or low charged ions by dissociating the molecule while the subsequent intense XUV pulse produces the high charge states. Thus, depending on whether the IR comes before of after the XUV pulse, we create a situation which allows us to compare the response of isolated atoms or ions with that of a molecule irradiated by exactly the same intense XUV pulse.

Our findings are summarized in Fig. 8, where the yield differences between the two situations IR-early and XUV-early are shown for all coincidence channels $(Q 1$, Q2). The region of low charge states is clearly dominated by a preceding XUV pulse. Asymmetric and very high charge states are produced more effectively if the IR laser arrives first and dissociates the molecule prior to the removal of additional electrons by the XUV.

In conclusion, we reached the highest charge states in our experiment after dissociation of iodine into separated atoms or ions. This is in contrast to observations with large Xe clusters, where a significant enhancement of XUV absorption was found in comparison to monomers due to collective effects that contribute to cluster ionization. ${ }^{32}$ For a system with only two constituents, like the $\mathrm{I}_{2}$ molecule, such collective phenomena are not active, and the more intuitive picture of individual atoms is still valid.

\section{Acknowledgements}

We acknowledge technical support from B. Knape and C. Kaiser, and we thank the scientific and technical team at FLASH for optimal beam conditions. M. Küb. and M. F. K. acknowledge support by the DFG via the Cluster of Excellence: Munich Center for Advanced Photonics. Y. H. J. is grateful for support from the NSFC, the National Basic Research Program of China, and the Shanghai Pujiang Program. A. R. acknowledges the support from Chemical Sciences, Geosciences, and Biosciences Division, Office of Basic Energy Sciences, Office of Science, U.S. Department of Energy, and from Kansas NSF EPSCoR "First Award” program.

\section{References}

1 G. Doumy, et al., Nonlinear Atomic Response to Intense Ultrashort X Rays, Phys. Rev. Lett., 2011, 106, 083002.

2 M. Richter, et al., Extreme Ultraviolet Laser Excites Atomic Giant Resonance, Phys. Rev. Lett., 2009, 102, 163002.

3 B. Rudek, et al., Resonance-enhanced multiple ionization of krypton at an Xray free-electron laser, Phys. Rev. A: At., Mol., Opt. Phys., 2013, 87, 023413.

4 B. Rudek, et al., Ultra-efficient ionization of heavy atoms by intense X-ray freeelectron laser pulses, Nat. Photonics, 2012, 6, 858.

$5 \mathrm{H}$. Thomas, et al., Shell explosion and core expansion of xenon clusters irradiated with intense femtosecond soft X-ray pulses, J. Phys. B: At., Mol. Opt. Phys., 2009, 42, 134018. 
6 T. Gorkhover, et al., Nanoplasma dynamics of single large Xenon clusters irradiated with superintense X-ray pulses from the linac coherent light source free-electron laser, Phys. Rev. Lett., 2012, 108, 245005.

7 R. Neutze, et al., Potential for biomolecular imaging with femtosecond X-ray pulses, Nature, 2000, 406, 752.

8 L. Fang, et al., Multiphoton Ionization as a clock to Reveal Molecular Dynamics with Intense Short X-ray Free Electron Laser Pulses, Phys. Rev. Lett., 2012, 109, 263001.

9 B. Erk, et al., Inner-shell multiple ionization of polyatomic molecules with an intense X-ray free-electron laser studied by coincident ion momentum imaging, J. Phys. B: At., Mol. Opt. Phys., 2013, 46, 164031.

10 B. Erk, et al., Ultrafast Charge Rearrangement and Nuclear Dynamics upon Inner-Shell Multiple Ionization of Small Polyatomic Molecules, Phys. Rev. Lett., 2013, 110, 053003.

11 M. Krikunova, et al., Strong-field ionization of molecular iodine traced with XUV pulses from a free-electron laser, Phys. Rev. A: At., Mol., Opt. Phys., 2012, 86, 043430.

12 M. Krikunova, et al., Ultrafast photofragmentation dynamics of molecular iodine driven with timed XUV and near-infrared light pulses, J. Chem. Phys., 2011, 134, 024313.

$13 \mathrm{~J}$. Ullrich, et al., Recoil-ion and electron momentum spectroscopy: reaction microscopes, Rep. Prog. Phys., 2003, 66, 1463.

14 H. Redlin, et al., The FLASH pump-probe laser system: Setup, characterization and optical beamlines, Nucl. Instrum. Methods Phys. Res., Sect. A, 2011, 635, 88.

15 B. H. Boo and N. Saito, Dissociative multiple photoionization of Br2, IBr, and I2 in the VUV and X-ray regions: a comparative study of the inner-shell processes involving $\mathrm{Br}(3 \mathrm{~d}, 3 \mathrm{p}, 3 \mathrm{~s})$ and $\mathrm{I}(4 \mathrm{~d}, 4 \mathrm{p}, 4 \mathrm{~s}, 3 \mathrm{~d}, 3 \mathrm{p})$, J. Electron Spectrosc. Relat. Phenom., 2002, 127, 139.

$16 \mathrm{~J}$. Tremblay, et al., Photoelectron spectroscopy of atomic iodine produced by laser photodissociation, Phys. Rev. A: At., Mol., Opt. Phys., 1988, 38, 3804.

$17 \mathrm{H}$. Kjeldsen, et al., Absolute photoionization cross sections of $\mathrm{I}+$ and $\mathrm{I} 2+$ in the 4d ionization region, Phys. Rev. A: At., Mol., Opt. Phys., 2000, 62, 020702.

18 A. Kramida, Y. Ralchenko, J. Reader, and NIST ASD Team(2013), NIST Atomic Spectra Database (ver. 5.1), Available: http://physics.nist.gov/asd.

19 D. Edvardsson, et al., An experimental and theoretical investigation of the valence double photoionisation of the iodine molecule, Chem. Phys., 2006, 324, 674 .

20 L. Fang, et al., Double Core-Hole Production in N2: Beating the Auger Clock, Phys. Rev. Lett., 2010, 105, 083005.

21 L. J. Frasinski, et al., Dynamics of Hollow Atom Formation in Intense X-Ray Pulses Probed by Partial Covariance Mapping, Phys. Rev. Lett., 2013, 111, 073002.

$22 \mathrm{~J}$. H. Posthumus, et al., Field-ionization, Coulomb explosion of diatomic molecules in intense laser fields, J. Phys. B: At., Mol. Opt. Phys., 1996, 29, 5811.

23 D. M. Villeneuve, M. Y. Ivanov and P. B. Corkum, Enhanced ionization of diatomic molecules in strong laser fields: A classical model, Phys. Rev. A: At., Mol., Opt. Phys., 1996, 54, 736.

24 T. Zuo and A. D. Bandrauk, Charge-resonance-enhanced ionization of diatomic molecular ions by intense lasers, Phys. Rev. A: At., Mol., Opt. Phys., 1995, 52, R2511. 
25 E. Constant, H. Stapelfeldt, and P. Corkum, Enhanced Ionization of Molecular Ions in Intense Laser Fields: Experiments on the Iodine Melecule, in Ultrafast Phenomena $X$, ed. P. Barbara et al., Springer Series in Chemical Physics. Springer, Berlin Heidelberg, 1996, vol. 62, pp. 105-106.

26 G. N. Gibson, et al., Direct evidence of the generality of charge-asymmetric dissociation of molecular iodine ionized by stronglaser fields, Phys. Rev. A: At., Mol., Opt. Phys., 1998, 58, 4723.

27 L. J. Frasinski, et al., Femtosecond dynamics of multielectron dissociative ionization by use of a picosecond laser, Phys. Rev. Lett., 1987, 58, 2424.

28 K. Codling, L. J. Frasinski and P. A. Hatherly, On the field ionisation of diatomic molecules by intense laser fields, J. Phys. B: At., Mol. Opt. Phys., 1989, 22, L321.

29 D. Normand and M. Schmidt, Multiple ionization of atomic and molecular iodine in strong laser fields, Phys. Rev. A: At., Mol., Opt. Phys., 1996, 53, R1958.

30 T. Seideman, M. Y. Ivanov and P. B. Corkum, Role of Electron Localization in Intense-Field Molecular Ionization, Phys. Rev. Lett., 1995, 75, 2819.

31 S. Chelkowski and A. D. Bandrauk, Two-step Coulomb explosions of diatoms in intense laser fields, J. Phys. B: At., Mol. Opt. Phys., 1995, 28, L723.

$32 \mathrm{H}$. Wabnitz, et al., Multiple ionization of atom clusters by intense soft X-rays from a free-electron laser, Nature, 2002, 420, 6915. 